\title{
The more the better - polyandry and genetic similarity are positively linked to reproductive success in a natural population of terrestrial salamanders (Salamandra salamandra)
}

\author{
BARBARA A. CASPERS,$*$ E. TOBIAS KRAUSE, ${ }^{*}$ RALF HENDRIX,$*+$ MICHAEL KOPP,+ \\ OLIVER RUPP,§ KATRIN ROSENTRETER* and SEBASTIAN STEINFARTZ* \\ *Department of Animal Behaviour, Bielefeld University, Morgenbreede 45, 33615 Bielefeld, Germany, †Max Planck Institute for \\ Evolutionary Biology, August-Thienemann-Str. 2, 24306 Plön, Germany, $₫$ Evolutionary Biology and Modelling Group, \\ Aix-Marseille University, LATP UMR-CNRS 7353, 13331 Cedex 3, Marseille, France, §Computational Genomics, Center for \\ Biotechnology (CeBiTec), and Cell Culture Technology, Bielefeld University, 33594 Bielefeld, Germany, |Zoological Institute, \\ Department of Evolutionary Biology, Technische Universität Braunschweig, Mendelssohnstr. 4, 38106 Braunschweig, Germany
}

\begin{abstract}
Although classically thought to be rare, female polyandry is widespread and may entail significant fitness benefits. If females store sperm over extended periods of time, the consequences of polyandry will depend on the pattern of sperm storage, and some of the potential benefits of polyandry can only be realized if sperm from different males is mixed. Our study aimed to determine patterns and consequences of polyandry in an amphibian species, the fire salamander, under fully natural conditions. Fire salamanders are ideal study objects, because mating, fertilization and larval deposition are temporally decoupled, females store sperm for several months, and larvae are deposited in the order of fertilization. Based on 18 microsatellite loci, we conducted paternity analysis of 24 female-offspring arrays with, in total, over 600 larvae fertilized under complete natural conditions. More than one-third of females were polyandrous and up to four males were found as sires. Our data clearly show that sperm from multiple males is mixed in the female's spermatheca. Nevertheless, paternity is biased, and the most successful male sires on average $70 \%$ of the larvae, suggesting a 'topping off' mechanism with first-male precedence. Female reproductive success increased with the number of sires, most probably because multiple mating ensured high fertilization success. In contrast, offspring number was unaffected by female condition and genetic characteristics, but surprisingly, it increased with the degree of genetic relatedness between females and their sires. Sires of polyandrous females tended to be genetically similar to each other, indicating a role for active female choice.
\end{abstract}

Keywords: female choice, heterozygosity, mating strategies, sperm competition, sperm staggering, spermatheca

Received 4 February 2013; revision received 9 September 2013; accepted 13 October 2013

Introduction

Males and females frequently employ differential mating strategies, which increase their own reproductive success

Correspondence: Barbara A. Caspers, Fax: + 49521 1062998;

E-mail: barbara.caspers@uni-bielefeld.de and Sebastian

Steinfartz, Fax: +49 391 8198; E-mail: s.steinfartz@tu-bs.de and reflect conflicting interests between the sexes. Sexual selection theory predicts that females gain reproductive success by choosing an optimal partner, whereas males should invest in a high number of mating opportunities (Trivers 1972). Nevertheless, females are often polyandrous and seek multiple matings with different males (Tregenza \& Wedell 1998, 2000; Jennions \& Petrie 2000; Garant et al. 2001; Tennessen \& Zamudio 2003), and they 
do so even though multiple mating has its costs, such as an increased risk of predation (Daly 1978; Holland \& Rice 1999) and/or a potential risk of sexually transmitted diseases (Sheldon 1993; Thrall et al. 1997). In general, multiple matings might provide polyandrous females with either direct or indirect benefits. Direct benefits are those that increase the expected number of offspring, for example due to male-provided resources, increased fertilization success or decreased risk of genetic incompatibility (Zeh \& Zeh 1997; Tregenza \& Wedell 1998, 2000, 2002). Indirect benefits, in contrast, increase offspring fitness (reviewed in Slatyer et al. 2012) or genetic variability (e.g. bet-hedging, Slatkin 1974).

Following multiple copulations, the sperm of different males may interact directly or indirectly, leading to important postcopulatory selection processes, such as sperm competition (Parker 1998; Wigby \& Chapman 2004) and cryptic female choice (Birkhead \& Pizzari 2002). These selection processes should be particularly intensive in species where females store the sperm of several males internally, with the outcome of selection partially depending on anatomical details of the female reproductive tract.

Tailed amphibians, such as salamander and newt species belonging to the suborder Salamandroidea, are well-suited for studying the effects of polyandry in the presence of intense sperm competition. Females from these species possess internal receptive organs (called spermathecae) that enable long-term sperm storage (Sever 2002), and for all species of that suborder investigated so far, paternity analysis has revealed polyandrous mating patterns for females (Jones et al. 2002; Garner \& Schmidt 2003; Tennessen \& Zamudio 2003; Myers \& Zamudio 2004; Steinfartz et al. 2006; Chandler \& Zamudio 2008). Previous studies in newts have revealed species-specific patterns of sperm storage with different consequences for the outcome of sperm competition and subsequent fertilization of ova. For the common newt, Lissotriton vulgaris, it has been suggested that the sperm of different males is staggered (layered) in the female's spermatheca and that the majority of eggs are fertilized by the last mating partner (so called 'last-male advantage'; Gabor \& Halliday 1997; Sever et al. 1999, but see Jehle et al. 2007). In contrast, for newts of the genus Taricha, laboratory studies indicate that the sperm of several males is mixed and 'topped off' in the spermatheca (i.e. only part of the second male's sperm can enter the spermatheca), leading to a pattern of first-male advantage (Jones et al. 2002). Sperm mixing, as opposed to staggering, should increase the potential of active competition among sperm from different males, but also for cryptic female choice.

In this study, we investigate the patterns and consequences of a polyandrous mating system under full natural conditions in the fire salamander (Salamandra salamandra), a terrestrial member of the Salamandroidea. In Central Europe, females of this species have recently been shown to be polyandrous, with up to three sires under natural conditions (Steinfartz et al. 2006). Mating occurs the year before the female gives birth to between 30 and 50 fully developed larvae, which are deposited in streams and ponds during the spring season. By collecting females prior to their deposition of larvae, complete female-offspring arrays sired under natural conditions can be analysed for concrete paternity patterns with high accuracy. Furthermore, in this species, larvae are deposited in the order in which they have been fertilized, which provides insights into the patterns of sperm storage. Therefore, this system offers a unique opportunity to analyse the effects of polyandry in terms of postcopulatory selection processes in a terrestrial vertebrate species under natural conditions (see Fig. 1).

For our study, we analysed 24 distinct mother-offspring arrays collected from nature, which resulted in more than 600 larvae, for which paternal genotypes were reconstructed based on 18 microsatellite loci. Our study design allowed us to estimate the degree of polyandry (i.e. the number of sires) under natural conditions and to analyse whether the observed paternity patterns are more likely explained by sperm mixing or staggering. In addition, we tested a variety of hypotheses concerning potential benefits of polyandry for females. To investigate direct benefits, we analysed whether female reproductive success (total number of deposited larvae) is influenced by the number of sires and/or by genetic characteristics of males, such as genetic similarity to females. To account for indirect benefits, we analysed the effects of polyandry on several correlates of offspring fitness, in particular offspring condition (size/weight at birth) and genetic variability (heterozygosity and internal relatedness). Body size at metamorphosis in amphibians is generally considered to be positively correlated with fitness (e.g. Semlitsch et al. 1988; Altwegg \& Reyer 2003), and size and weight at birth are positively correlated with survival to and size at metamorphosis in the fire salamander (Alcobendas et al. 2004). Therefore, females may gain indirect benefits from polyandry if sperm competition favours males that sire larger/heavier offspring. Furthermore, genetic variability (heterozygosity) has been shown to correlate with larval growth rates (Rowe et al. 1999) and is generally assumed to increase fitness (reviewed in Hansson \& Westerberg 2002). Thus, polyandrous females might have more heterozygous offspring than monogamous females if they choose extra mates that are genetically different from themselves.

In summary, our study used high-resolution genetic paternity analysis to investigate potential direct or 


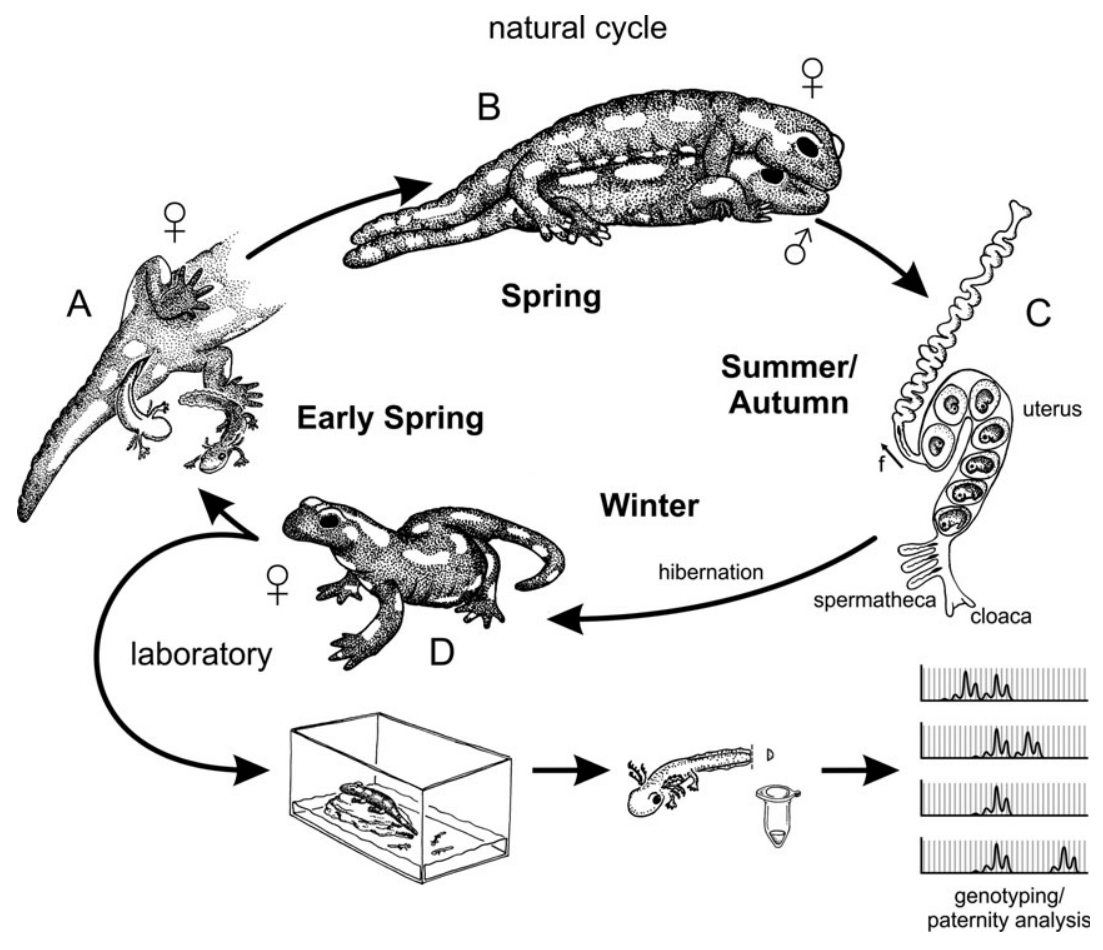

Fig. 1 The natural reproductive cycle of the fire salamander (Salamandra salaman$d r a)$ in Central Europe in context of the underlying design of our study. (A) Females deposit larvae into aquatic habitats in early spring. (B) Four to six weeks after the deposition of larvae, females mate on land (occasionally with multiple males) and store the sperm in their spermatheca. (C) Eggs are fertilized in the order of their appearance in the upper part of the oviduct during summer. Fertilized embryos mature over winter until the next spring. (D) Gravid females were collected on their way to the larval habitats and brought to the laboratory. Female-offspring arrays were collected and used for paternity analysis (modified after Greven 1998). indirect benefits of polyandry in a terrestrial vertebrate in which females were fertilized under completely natural conditions.

\section{Material and methods \\ General study design and origin of female salamanders}

Our study population is located in the Kottenforst near Bonn (Germany), which is a continuous, old, deciduous forest representing a typical Central European habitat of Salamandra salamandra. In this population, females migrate in early spring (usually by the end of March) to small first-order streams or stagnant water bodies (e.g. small pools, ditches and tyre ruts), where they deposit their larvae (Steinfartz et al. 2007 and Fig. 1A). Several weeks after this deposition (around mid-April to mid-May), females mate with one or several males and store the sperm in their spermatheca (Fig. 1B,C). Several months later, the sperm is activated to swim up the oviduct and fertilize eggs that have entered the upper part of the uterus (Fig. 1C). Importantly, eggs are fertilized in the temporal order of their appearance in the uterus and remain in this order for further development. As a consequence, larvae are deposited in the order they were sired (Fig. 1C). By collecting gravid females on their way to the larval habitats and keeping them in the laboratory until deposition of their larvae, it is easy to sample comparably large numbers of female-offspring arrays that reflect mating patterns and order of fertilization from natural conditions (e.g. Jones et al. 2002; see Fig. 1).

With the permission of the nature reserve authority of the Stadt Bonn, we collected 40 potentially pregnant female fire salamanders on their way to the deposition habitats in the Kottenforst during rainy nights in spring 2010. Immediately after capture, females were measured for their mass and body size (snout-ventlength; SVL) and kept in the laboratory until they had deposited all of their larvae. Females were housed individually in transparent plastic fauna boxes $(41 \mathrm{~cm} \times$ $14 \mathrm{~cm} \times 18 \mathrm{~cm}$ ) that were equipped with flat stones, bark and moss to provide dry hiding places, as well as with a 2- to 4-cm layer of water for the deposition of larvae (see Caspers \& Steinfartz 2011 for further details).

\section{Sampling of larvae and females}

Of the 40 collected females in the wild, 24 females deposited their larvae in the laboratory. The resultant female-offspring arrays were used for further analysis. As females often deposit larvae during the night, we collected larvae each morning and grouped them into batches, that is, larvae from one female and one night were grouped together and treated as a single batch. Each newly born larva was weighed (Sartorius BL150S balance accuracy $\pm 0.001 \mathrm{~g}$ ) and measured (SVL, millimetre paper, accuracy $\pm 0.5 \mathrm{~mm}$ ). Subsequently, the larvae were kept in their respective batches and fed once a 
week with Chironomus larvae ad libitum. After the larvae had reached a minimum size of approximately $5 \mathrm{~cm}$ in length, we took a small tissue sample from the back flipper for DNA extraction and further genetic analyses. Four weeks after the deposition of the last larva, females were sampled by clipping a single tip of a toe. Total genomic DNA was extracted from adult and larval tissue samples using the NucleoSpin Tissue kit (Macherey-Nagel).

\section{Microsatellite loci and paternity analysis}

All 24 mother-offspring arrays were genotyped for 18 S. salamandra-specific polymorphic microsatellite loci, as described previously (Steinfartz et al. 2004; Hendrix et al. 2010). The details of the PCR-multiplex combinations of loci and specific amplification procedures are given in Data S1 (Supporting Information). We used the program GERUD2.0 (Jones 2005) to reconstruct potential paternal genotypes for each female-offspring array. As the current version of GERUD2.0 can only handle a maximum of 10 loci at a time, we analysed the genotypes of potential fathers by running different combinations of loci for the same female-offspring array. To make use of the full set of 18 loci, we used up to three different combinations of loci, such that at least three loci differed and at least three loci overlapped between combinations. GERUD2.0 infers all minimum-father combinations for a given offspring array and ranks these combinations based on patterns of Mendelian segregation and genotype frequencies found in the original population, which were estimated from 1100 adult salamanders collected in a parallel study across the whole Kottenforst; see Data S2 (Supporting Information). A minimum-father combination is defined as a set of fathers of minimal size needed to explain paternity in a specific offspring array. If GERUD 2.0 suggested multiple sires, we used the most likely minimum-father combination to assign paternal genotypes to each larva across all 18 loci (exclusion probabilities for all 18 loci were $>0.99$, see Data S3; Supporting Information). For example, if GERUD2.0 suggested two distinct paternal genotypes $\mathrm{AC}$ and $\mathrm{BC}$ at a given locus, with the female genotype at this locus being $\mathrm{AC}$ and that of the larva $\mathrm{AB}$, then clearly, the only admissible paternal genotype is BC. To decrease the potential of genotyping errors, fathers that had sired only a single larva were accounted for only when they differed from other fathers at least at two distinct loci, leading to the exclusion of two paternal genotypes in the whole data set. In eight of nine cases with multiple paternity, we were able to assign each larva unambiguously to one paternal genotype, with the exception of four larvae from female F_55_10, which were discarded from further analysis.
For female F_52_10, more than ten larvae could not be unambiguously assigned, so we discarded this femaleoffspring array from the sperm-pattern analyses. We performed several in-depth statistical tests to rule out potential biases in the paternity analysis, which could arise because the number of sires was estimated from offspring arrays of varying size (see Data S4, Supporting Information).

Based on the maternal and reconstructed paternal genotypes, we calculated an allele-sharing matrix using the Microsatellite tOOLKIT (version 3.1.1; Park 2001), which allowed to test whether the degree of genetic similarity, that is, the proportion of shared alleles, between parents had an impact on the number of deposited larvae.

\section{Analysis of sperm storage/fertilization patterns}

As females of S. salamandra can store and use the sperm from multiple males for fertilization, we explored whether the sperm in the female's spermatheca was mixed or staggered (see also Introduction). As larvae are deposited in the temporal order of their fertilization (see above and Fig. 1), the paternity analysis of batches of larvae allows direct insight into sperm storage patterns.

To investigate whether mixing or staggering of sperm is more likely in fire salamanders, we conducted a permutation test with mixing as the null hypothesis, using data from females that deposited at least two batches of offspring sired by at least two different males. Under the mixing hypothesis, offspring from different fathers should be distributed randomly across batches, that is, 'father' and 'batch' should be independently random variables. We tested this hypothesis using the following steps: First, we calculated for each offspring array a measure of association between 'father' and 'batch'. Because 'father' is a nominal variable, whereas 'batch' is ordinal, we used a measure of nominal-ordinal association proposed by Agresti (1981). We note that we obtained very similar results when using the more widely known Cramér's V, which discards, however, the information about the order of batches. For two fathers, Agresti's $\Delta$ is given by the probability that a randomly chosen offspring from father 1 is deposited in a later batch than a randomly chosen offspring from father 2, minus the probability that an offspring from father 1 is deposited in an earlier batch than an offspring from father 2. For more than two fathers, $\Delta$ is a weighted average over the pairwise measures. We then calculated $\bar{\Delta}$ as the mean value of $\Delta$ over all females. Subsequently, we estimated the distribution of $\bar{\triangle}$ under the mixing hypothesis, by randomly reshuffling the offspring over the batches of their respective mothers, calculating $\bar{\triangle}$ and repeating this procedure 100000 times. 
Finally, a $P$-value was obtained by comparing this distribution to the $\bar{\triangle}$ from the original data. To evaluate the power of this method, we also calculated the distribution of $\bar{\triangle}$ under various degrees of staggering. This was achieved by first re-ordering the offspring from each female according to their fathers (with the order of fathers being chosen at random) and then reshuffling a randomly chosen subset of $q=0,25,50$ or $75 \%$ of the offspring (0\% reshuffling corresponds to perfect staggering, whereas $100 \%$ reshuffling would recover the mixing model).

\section{Statistical analysis of the potential benefits of polyandry}

Direct benefits: We tested for potential direct benefits of polyandry for female reproductive success by analysing the number of larvae deposited per female using a general linear model (GLM) with minimal number of fathers (as determined from the paternity analysis), mean proportion of alleles (MNA) shared by motherfather pairs (Bowcock et al. 1994), maternal mass, maternal size and maternal observed heterozygosity $\mathrm{HZ}_{\mathrm{obs}}$ as explanatory variables. $\mathrm{HZ}_{\mathrm{obs}}$ was determined using the IRmacro (Amos et al. 2001).
Indirect benefits: Statistical analysis of the effects of polyandry on larval size and mass at deposition, as well as on larval observed heterozygosity $\mathrm{HZ}_{\mathrm{obs}}$ and internal relatedness (IR, Amos et al. 2001) are presented in Data S5 and S6, respectively (Supporting Information).

To analyse genetic diversity among the partners of polyandrous females, we used an analysis of similarity (ANOSIM; Primer 6, Primer-E ltd. Plymouth, UK) with the females' IDs as the grouping factor. ANOSIM is used to statistically determine whether individuals within a priori defined groups are more similar to each other than individuals among groups (Clark \& Warwick 1994). Unless otherwise stated, statistical analyses were performed using IBM SPSS STATISTICS 20.

\section{Results}

\section{Paternity analysis and observed patterns of female sperm storage}

In total, we analysed 603 larvae deposited by 24 females. Females deposited an average of $25.13 \pm 13.36$ (SD) larvae, with a minimum of four and a maximum of 44 (see Table 1). The number of batches per female varied from 1 to 10 , with a median of three. Paternity

Table 1 Larval deposition data of the 24 female fire salamanders from this study. The last column shows the probabilities that a female had more mates than sires, as calculated in Data S4 (Supporting information)

\begin{tabular}{|c|c|c|c|c|c|}
\hline Female ID & $\begin{array}{l}\text { Number of deposition } \\
\text { days/nights }\end{array}$ & $\begin{array}{l}\text { Minimal number } \\
\text { of males }\end{array}$ & $\begin{array}{l}\text { Number of } \\
\text { deposited larvae }\end{array}$ & $\begin{array}{l}\text { Number of unfertilized } \\
\text { eggs/nonviable embryos }\end{array}$ & $\begin{array}{l}\text { Probability of more } \\
\text { mates than sires }\end{array}$ \\
\hline F 02-09 & 2 & 1 & 33 & 1 & $<0.0001$ \\
\hline F 03-09 & 3 & 1 & 9 & 0 & 0.0178 \\
\hline F $05-10$ & 2 & 1 & 29 & 0 & $<0.0001$ \\
\hline F 10-09 & 1 & 1 & 17 & 0 & 0.001 \\
\hline F $12-10$ & 1 & 1 & 4 & 0 & 0.0995 \\
\hline F $27-10$ & 3 & 1 & 7 & 0 & 0.0357 \\
\hline F $31-10$ & 5 & 1 & 40 & 1 & $<0.0001$ \\
\hline F $42-10$ & 2 & 1 & 13 & 1 & 0.0043 \\
\hline F 43-10 & 6 & 1 & 28 & 4 & $<0.0001$ \\
\hline F $49-10$ & 10 & 1 & 34 & 7 & $<0.0001$ \\
\hline F $50-10$ & 8 & 1 & 30 & 12 & $<0.0001$ \\
\hline F $58-10$ & 6 & 1 & 18 & 1 & 0.0007 \\
\hline F $60-10$ & 9 & 1 & 22 & 2 & 0.0002 \\
\hline F $63-10$ & 1 & 1 & 10 & 0 & 0.0125 \\
\hline F $67-10$ & 3 & 1 & 4 & 0 & 0.0995 \\
\hline F $33-10$ & 5 & 2 & 15 & 2 & 0.0148 \\
\hline F $48-10$ & 6 & 2 & 27 & 5 & 0.0299 \\
\hline F 73-10 & 1 & 2 & 42 & 0 & 0.0299 \\
\hline F 09-09 & 2 & 3 & 35 & 0 & 0.0233 \\
\hline F $13-10$ & 3 & 3 & 44 & 3 & $<0.0001$ \\
\hline F $52-10$ & 7 & 3 & 40 & 1 & $<0.0001$ \\
\hline F $55-10$ & 5 & 3 & 25 & 1 & 0.0019 \\
\hline F $02-10$ & 4 & 4 & 34 & 0 & 0.0045 \\
\hline F 05-09 & 3 & 4 & 44 & 0 & 0.0011 \\
\hline
\end{tabular}


analysis, based on microsatellite loci of larvae and mothers, revealed that nine of 24 female-offspring arrays $(37.5 \%)$ had multiple sires, whereas the majority of arrays $(62.5 \%)$ had only one sire. Out of the polyandrous females, three females mated with at least two different males (33\%), four females mated with at least three different males (45\%) and two females (22\%) mated with at least four different males (see Table 1). For eight of the nine female-offspring arrays with multiple sires, we were able to unambiguously assign each larva to one of the potential paternal genotypes (Fig. 2A). The proportion of paternity was highly skewed, with the most successful male siring on average $70 \%$ of the offspring (chi-square test; $\chi_{21}^{2}=62.27$, $P<0.0001$; see Fig. 2A).

As expected from the particular fertilization mechanism in female fire salamanders, in which eggs are fertilized in order of their appearance in the uterus (see Fig. 1), mass and total size of the larvae decreased with their date of deposition (Data S5, Supporting Information). To analyse whether the sperm of different males was mixed or staggered, we analysed the association between 'father' and 'batch' for the seven females that deposited at least two batches of offspring sired by at least two different males. These females had a $\Delta$-coefficient of $0.175 \pm 0.11$ (mean \pm SD), which is fully compatible with the mixing hypothesis $(P>0.873$; see Fig. 2B). Furthermore, we found that our statistical approach had a high power to detect staggering combined with up to $50 \%$ of mixing. Thus, our analyses suggest that the sperm of different males is mixed rather than staggered in the females' spermatheca prior to fertilization.

\section{Potential benefits of polyandry}

We found a strong and significant positive relationship between the minimal number of fathers and female reproductive success, as measured by the number of deposited larvae (GLM; factor: minimal number of fathers: $F_{1,18}=11.35, P=0.003$, see Table 2; Fig. 3). As illustrated in Fig. 3, the main cause for this relationship is that the minimal number of larvae per female increases with the number of sires, whereas monogamous females deposited between four and 40 larvae, females with two sires had between 15 and 42 larvae, females with 3 sires between 25 and 44 larvae, and females with four sires between 34 and 44 larvae. Intriguingly, an inverse, though nonsignificant, pattern can be seen for the number of unfertilized eggs/nonviable embryos, whose maximal number decreases with the number of sires (see Data S7, Figure S7b, Supporting Information). These data, however, should be interpreted with caution, because we do not know to what
(A)
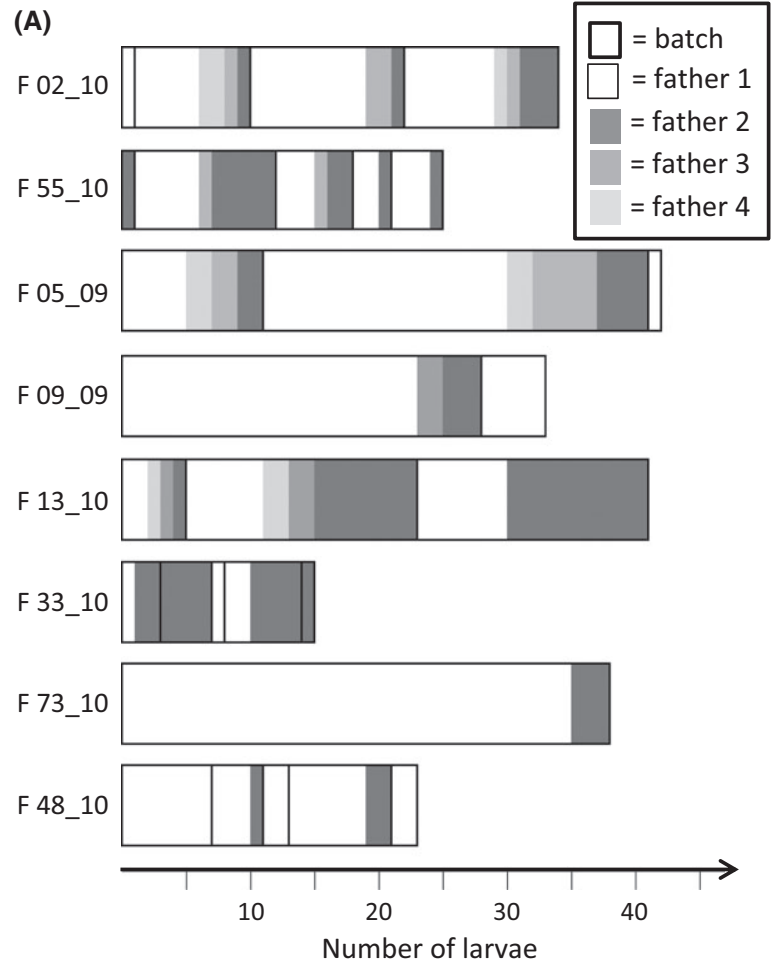

(B)

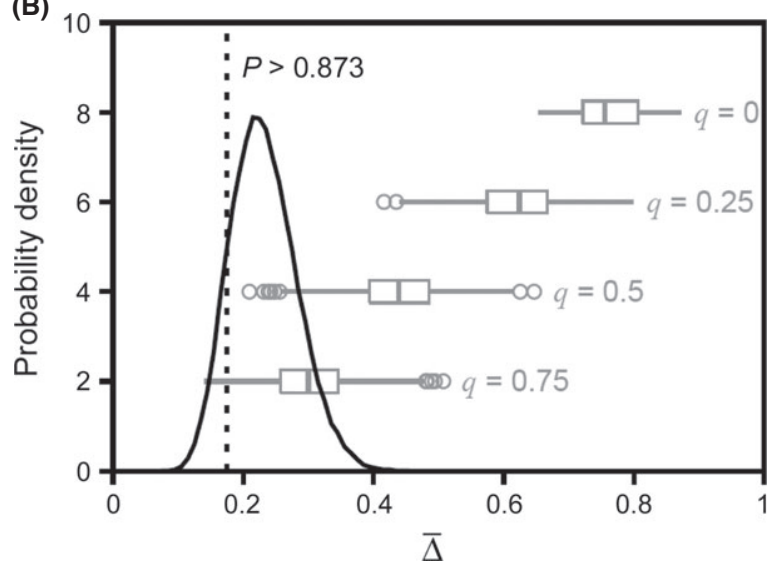

Fig. 2 Sperm storage patterns in Salamandra salamandra. (A) Visual representation of the genotyped offspring arrays from polyandrous females. Different grey scales indicate larvae sired by different fathers. Thick lines show the grouping of larvae into batches deposited during different nights. (B) Statistical analysis of fertilization patterns, using Agresti's (1981) $\Delta$-statistic as a measure of association between father and batch in the offspring of polyandrous females. The vertical dotted line marks the value of $\bar{\triangle}$; observed in the data. The solid curve shows the frequency distribution of $\bar{\triangle}$; under the null hypothesis of sperm mixing, estimated from 100000 permutations of the original data (histogram with class width 0.01 ). The $P$-value is the proportion of this distribution above the measured value. Boxplots show the distribution of the test statistic under the stratification hypothesis combined with $100 * q$ per cent mixing (1000 permutations of the original data). The variation in the absence of mixing $(q=0)$ stems from the different configurations in which the offspring can be stratified. 
Table 2 Analyses of direct benefits of polyandry (number of deposited larvae) in female fire salamanders as a function of the number of reconstructed sires

\begin{tabular}{lrllll}
\hline & \multicolumn{4}{l}{$\begin{array}{l}\text { Number of } \\
\text { larvae deposited by } \\
\text { females }\end{array}$} & \\
\cline { 2 - 6 } & $F$ & d.f. & $P$ & $*$ \\
\hline Minimal number of father & 11.35 & 1,18 & $\mathbf{0 . 0 0 3}$ & + \\
Maternal-paternal shared alleles & 7.67 & 1,18 & $\mathbf{0 . 0 1 3}$ & + \\
Maternal mass & 1.97 & 1,18 & 0.18 & \\
Maternal size & 0.30 & 1,18 & 0.59 & \\
Maternal heterozygosity & 0.05 & 1,18 & 0.83 & \\
$R^{2}$ of the model & & & 0.56 & \\
\hline
\end{tabular}

*Direction of effect: + indicates a positive relationship. significant results are given in boldface

degree unfertilized eggs can be reabsorbed by the female organism.

We observed a large variation in the number of deposited larvae. Females of Salamandra salamandra typically develop between 30 and 50 ova (Thiesmeier \& Grossenbacher 2004), which coincides well with the maximal number of deposited larvae observed here. Females with a small number of larvae might have had a low fertilization success and reabsorbed the majority of ova. Alternatively, we cannot exclude that some females already had deposited part of their larvae before being caught in the wild and brought to the laboratory. By itself, this possibility should be independent of the number of sires (and other variables) and, hence, not cause a bias in our analysis. However, problems may arise if the number of sires/mating partners is

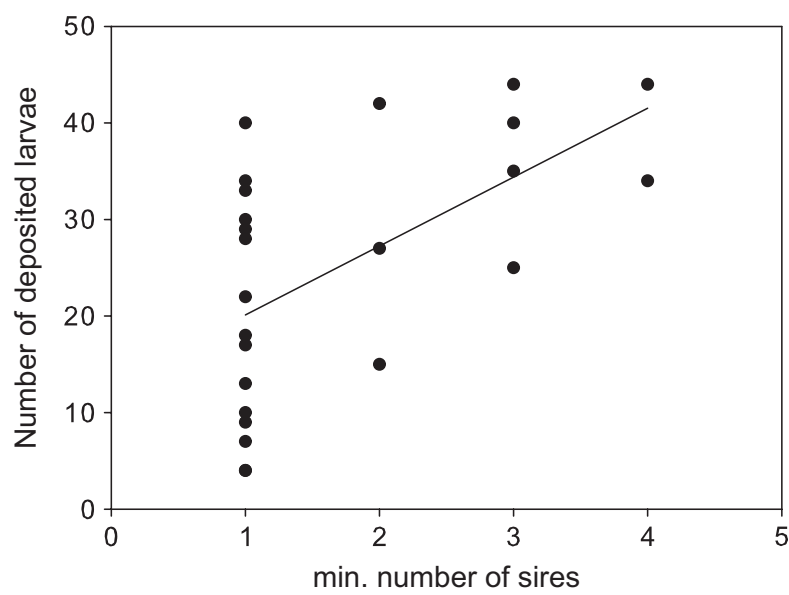

Fig. 3 Positive relationship between reproductive success (indicated by the number of deposited larvae) and the level of polyandry (i.e. the minimal number of sires) per female as shown by the solid line (general linear model, factor number of sires: $P=0.003 ; R^{2}$ of the entire model $=0.56$ ). For the full model see Table 2 . more likely to be underestimated in small than in large offspring arrays (i.e. some of the data points in the lower left corner of Figure 3 would need to be shifted to the right). In Data S4 (Supporting Information), we present additional statistical analyses indicating that such bias is unlikely to have had a significant impact on our analysis. In addition, the relationship between reproductive success and number of sires remains significant when the four females that deposited $<10$ larvae are excluded from the analysis (data not shown).

Furthermore, we found a significant influence of genetic similarity between mother-father pairs on the number of larvae deposited by a female, indicating that reproductive success is positively correlated with parental genotype similarity (Table 2, Fig. 4). As illustrated by Figure 4, this relationship is mainly due to the data from monogamous females, that is, it is independent of the effect of polyandry. Incidentally, parental similarity is also positively (though not significantly) correlated with the number of unfertilized eggs or dead-born larvae (see Data S7, Figure S7a, Supporting Information), but this result might be spurious, because the number of reabsorbed ova is unknown. On the other hand, reproductive success was not influenced by female quality, as neither female mass, female size nor observed female heterozygosity affected the number of deposited larvae (see Table 2).

To test for indirect benefits of multiple mating, we analysed whether female polyandry influenced various measures of offspring quality. Mean size and mass of

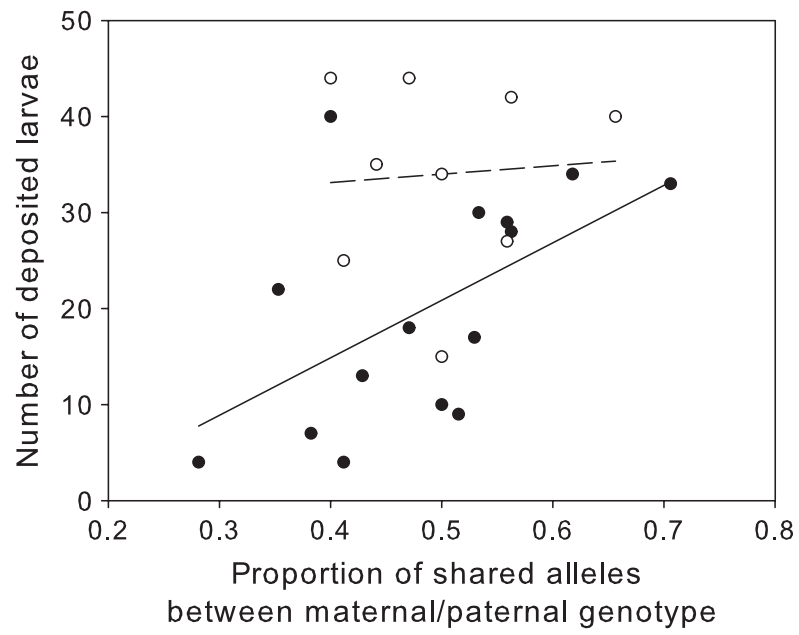

Fig. 4 The relationship between parental relatedness and reproductive success. The factor 'allele sharing between mother and father' had a significant positive impact in the general linear model on the number of larvae (Tab. 3). Here, the solid line indicates the significant relationship for monogamous females (filled dots; $n=15 ; \mathrm{r}_{\mathrm{P}}=0.55, P=0.032$ ) and the dashed line the less pronounced and nonsignificant relationship for polyandrous females (open dots; $n=9 ; r_{\mathrm{P}}=0.07, P=0.86$ ). 
larvae per offspring array were positively correlated with maternal mass, but independent of the minimal number of fathers and the degree of parental similarity (see Data S5, Supporting Information). In addition, we did not find any differences in average offspring heterozygosity between polyandrous and monogamous females, nor was there an effect on larval internal relatedness (see Data S6, Supporting Information). However, as expected, polyandry increased genetic diversity among larvae, as the mean number of alleles per locus (MNA) was significantly higher in offspring arrays of polyandrous as compared to monogamous females (t-test: $\mathrm{MNA}_{\text {polyandrous }}=3.0+0.23$ mean $\pm \mathrm{SD}$; $\mathrm{MNA}_{\text {monogamous }}=2.49+0.33$ mean $\pm \mathrm{SD} ; T_{1,22}=4.06$; $P<0.001)$. Nevertheless, polyandrous females do not appear to seek genetically dissimilar males. Indeed, males that sired the offspring of a specific polyandrous female were, on average, more genetically similar to each other than sires of different females (ANOSIM Global $R=0.531, P=0.001)$. It is important to mention that this pattern does not depend on genetic differentiation between sampling locations (ANOSIM with location as grouping variable is non-significant; Global $R=0.002, P=0.42$, see Fig. 5).

\section{Discussion}

We have used high-resolution genetic analysis combined with a special sampling design to investigate patterns and consequences of multiple paternity in a population of fire salamanders (Salamandra salamandra) under fully natural conditions, to an extent not previously achieved for a terrestrial vertebrate species. About one-third of the sampled females were polyandrous and carried larvae sired by up to four males (Table 1). We show that sperm from multiple males is mixed rather than staggered in the female's spermatheca (Fig. 2), which may have important consequences for processes and patterns of sexual selection. Our careful and unbiased paternity analysis revealed that multiple mating increases female reproductive success (i.e. the number of deposited larvae, Fig. 3). In contrast, we found no evidence that polyandry confers indirect genetic benefits, such as an increased offspring heterozygosity or a decreased internal relatedness. Indeed, our data suggest that female reproductive success is positively correlated with genetic similarity between females and sires (Fig. 4) and that polyandrous females mated with males that are genetically similar (rather than dissimilar) to each other (Fig. 5).

\section{Patterns of sperm storage and sperm use}

The outcome of sperm competition and sexual selection may depend on the pattern of sperm storage by females. Our paternity analysis showed that sperm of different males is mixed rather than staggered in the spermatheca of female fire salamanders and that approximately $70 \%$ of the larvae from polyandrous females are sired by a single male (see Fig. 2). Because we did not observe the matings with the different sires in nature, we can only speculate about their temporal order (i.e. whether firstor last-male advantage would apply), but our observations are more compatible with the 'topping off' mechanism suggested by Jones et al. (2002) for Taricha granulosa. According to this hypothesis, the sperm from the first partner is taken up completely, whereas the sperm from successive males is taken up only within the limits of the remaining space in the female's spermatheca. Subsequently, sperm from all males can mix freely, but the first male will fertilize the majority of eggs, simply because most of the sperm in the spermatheca originates from him. This interpretation is further strengthened by the fact that insemination in both fire salamanders and Taricha newts (Jones et al. 2002) requires the female to actively take up a spermatophore previously deposited by the male. Thus, males have no opportunity to influence sperm storage (as e.g. in
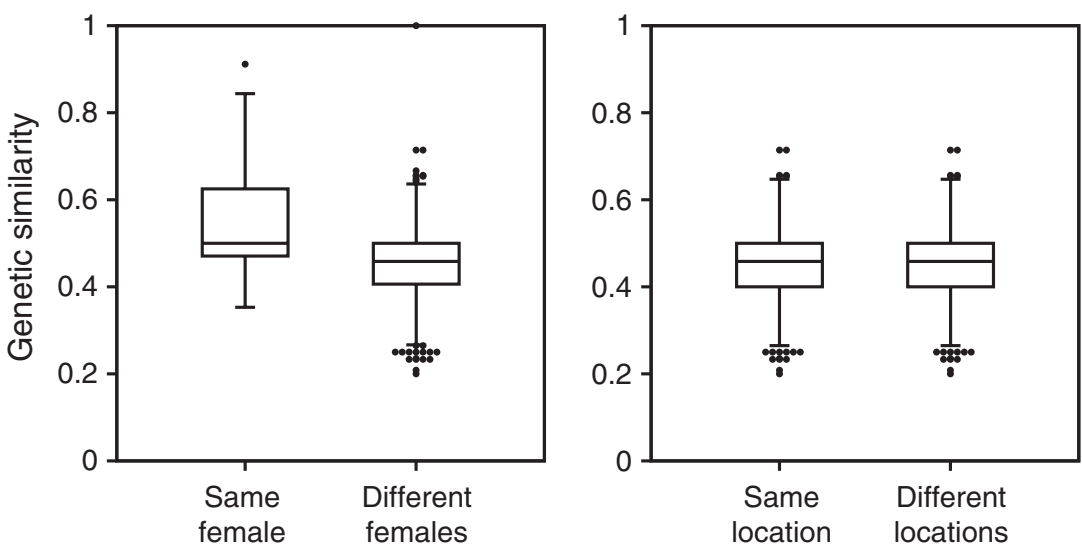

Fig. 5 Genetic similarities (proportion of shared alleles) between pairs of reconstructed males from the same or different females (left) or from the same and different sampling locations (right). 
odonates, where males can remove the sperm from previous partners; Waage 1979), and last-male advantage could only result from strong cryptic female choice.

The above argument does not imply, however, that the proportion of larvae sired per male is simply proportional to the amount of sperm in the spermatheca. Indeed, sperm mixing, as demonstrated in our study, sets the stage for competition between the sperm from different males, as well as for cryptic female choice. At the very least, sperm mixing ensures that females can use multiple mating as an insurance against inviable or incompatible sperm. Thus, in species where sperm is stored for a long time, such as in the fire salamander, sperm mixing may be an advantageous mechanism to increase the benefits of postmating intermale competition for the female. We turn to the discussion of these benefits next.

\section{Benefits and consequences of polyandry in the fire salamander}

Females may benefit from multiple mating by increasing their reproductive success (Zeh \& Zeh 1997; Tregenza \& Wedell 1998, 2000) or by gaining genetic benefits for their offspring (Madsen et al. 1992; Stapleton et al. 2007). Here, we have shown that, in fire salamanders, the degree of polyandry is positively correlated with the number of deposited larvae (Fig. 3), which fits well with other studies in which females were shown to increase their fertilization success by mating with multiple males (Tregenza \& Wedell 1998). In our study, the maximal number of larvae was independent of the number of mates (and probably close to the number of ova per female), but whereas monogamous females showed a large variance in reproductive success (between 4 and 40 larvae), none of the polygamous females had offspring numbers that were particularly low. Thus, multiple mating seems to ensure a high fertilization success. Since, to our knowledge, male fire salamanders do not provide any kind of resources to the female (unless females could gain energy by resorbing spermatophores), this increase in fertilization success may most likely be the result of a decreased risk of genetic incompatibility (Zeh \& Zeh 1997; Tregenza \& Wedell 1998, 2000; Dziminski et al. 2008). This interpretation is supported by our observation that the maximal number of unfertilized eggs decreased with the number of sires (Figure S7b, Supporting Information).

In contrast, we did not find any evidence for indirect benefits of multiple mating. Such indirect benefits, which increase offspring fitness, could arise if females accepted extra mates only when these exhibit particularly high quality or compatibility (e.g. Gabor \& Halliday 1997; Jehle et al. 2007). Here, we investigated whether the degree of polyandry influences two classes of potential correlates of offspring fitness, that is, size and weight at birth and mean offspring heterozygosity and internal relatedness. Size and weight at birth are positively related with fitness in fire salamanders (Alcobendas et al. 2004), but in our study, they did not show any dependence on the number of sires (Data S5, Supporting Information). Similarly, heterozygosity is often assumed to be advantageous in general (Amos et al. 2001; Hansson \& Westerberg 2002) and has been shown to correlate with larval growth rate in toads (Rowe et al. 1999). In the context of polyandry, offspring heterozygosity could increase with the number of sires if females accepted additional mates that are particularly genetically different from themselves. In our study, however, no such effect was found, as both offspring heterozygosity and internal relatedness were independent of the degree of polyandry.

\section{Genetic similarity and mate choice}

Our microsatellite loci data allowed us to determine the degree of genetic similarity between individuals. This analysis yielded two surprising results: First, males mated to a specific polyandrous female tend to be genetically similar among each other (Fig. 5). Second, and independent of polyandry, genetic similarity between mating partners is positively correlated with female reproductive success, despite considerable variation in the data (see Fig. 4)

The first result, that is, high genetic similarity between males mated to a single female, is in marked contrast to the common idea that females should increase genetic variability among their offspring by choosing extra mates that are genetically different from their principal or first mates, as has been shown, for example, in barn swallows (Stapleton et al. 2007), reed buntings (Sluter et al. 2007) and fur seals (Hoffman et al. 2007). The pattern found in our study would also suggest some form of female mate preference, favouring genetically similar, rather than dissimilar males. Accordingly, it is tempting to speculate that females prefer mates that are genetically similar to themselves. However, to firmly support such a conclusion, one would need additional data not available for this study. In particular, a comparison of genotypes of successful sires with the distribution of male genotypes in a specific sampling area would shed further light on potential selective mating in this system.

The second result suggests that genetic similarity between mating partners seems to increase fertilization success, probably by decreasing the risk of genetic incompatibilities. Studies showing a higher mating/ fertilization success with genetically similar males are, so far, quite rare. For Peron's tree frog, Litoria peronii, 
where fertilization is external, sperm competition experiments showed that genetically more similar mates fertilized more eggs (Sherman et al. 2008). Similar results were also found in arctic charr, Salvinus alpinus (Liljedal et al. 2008). In tiger salamanders (Ambystoma tigrinum), fertilization success is increased for males that are similar to females at the MHC complex, but in the same study no such correlation could be found for microsatellite loci (Bos et al. 2009). In contrast, in common newts (Lissotriton vulgaris), most eggs were fertilized by genetically dissimilar males under laboratory conditions (Jehle et al. 2007). All these studies, however, focused on sperm competition between males (potentially mediated by cryptic female choice), and it is unclear to what degree genetic similarity affects reproductive success of females.

The studies on Peron's tree frog (Sherman et al. 2008) and arctic charr (Liljedal et al. 2008) are, however, also interesting for another reason. These species coexist with closely related species or morphs, respectively, and the authors suggested that postcopulatory selection for genetically more similar mates might be a mechanism to avoid hybridization or extreme outbreeding, which would result in maladapted offspring. Indeed, the population of fire salamanders studied here is in the process of habitat-dependent adaptive divergence and shows considerable genetic differentiation between two ecologically differentiated types (with larval habitats in streams versus small temporary water bodies such as ponds, ditches and tire ruts; see Steinfartz et al. 2007). Although these ecotypes have not yet achieved the status of distinct species but are rather in an early stage of adaptive speciation, we might suspect that, also in this system, selection favours mating within, rather than between, differentially adapted types. As already mentioned above, we do not know whether females prefer males that are genetically more similar to themselves, but we may speculate that this is the case, and that they only mate with males that they have actively chosen before (including active take-up of the spermatophore). Indeed, anecdotal evidence from our own unpublished work suggests that female fire salamanders, although they had the opportunity to mate with two different males, fertilized their eggs with stored sperm from a previous year (Caspers, Krause pers. observation). This finding could indicate that females mate selectively and only accept males that meet their preferences. Strong selective mating might also explain why the proportion of multiple paternity found in our study is, with $37.5 \%$, clearly lower than that reported for other salamander species, such as $70 \%$ in the spotted salamander (Ambystoma maculatum, Myers \& Zamudio 2004), $85 \%$ in the red-backed salamander
(Plethodon cinerus, Liebgold et al. 2006) and 96\% in the Ocoee salamander (Desmognathus ocoee, Adams et al. 2005). If the hypothesis of selective mating holds true, both female preference for similar males and the correlation between parental similarity and reproductive success might be interpreted in the context of adaptive genetic divergence between subpopulations - and potentially, as a step towards ecological speciation. At the same time, however, our data also show that mating with males that are genetically too similar may come with costs of inbreeding, such as a higher proportion of unfertilized eggs and/or dead-born larvae (Data S7, Supporting Information). Thus, selection seems to balance between mating with mates of the own type and avoiding the costs of inbreeding.

\section{Conclusions}

In conclusion, we found that female fire salamanders are frequently polyandrous and that the sperm of several males is mixed in the female's spermatheca. Multiple mating seems to be beneficial for females primarily because it reduces the risk of low fertilization success (direct benefit). In contrast, we found no evidence that polyandry increases offspring fitness (indirect benefits).

Furthermore, and independent of the degree of polyandry, we found that female reproductive success increases if they mate with males that are genetically similar to themselves and that the partners of polyandrous females are genetically similar to each other. These results contrast with common assumptions concerning heterozygosity advantage, but they could be a consequence of the recent divergence of our fire salamander population and the selective forces that prevent individuals from outbreeding. Future investigations will need to study the relative importance of pre- and postmating mechanisms (mate preferences vs. sperm competition and cryptic female choice), and to what extent a loss of offspring heterozygosity entails relevant fitness costs.

\section{Acknowledgements}

We thank Annika Keller, Daniel Geller and Timm Reinhardt for collecting salamanders in the field and Elke Hippauf for support in the laboratory. Werner Jamin, Michael Meyerhoff and Jana Derbogen helped with maintenance of the salamanders and larvae. We are grateful to Adam Jones for providing us the source code of GERUDSIM3.0 for test statistics. This project was funded by a grant from the German Research Foundation (DFG) to B.A.C. (CA 889/1-1) and in part by a research grant from the Ethologische Gesellschaft for E.T.K., R.H. was supported by a doctoral stipend of the VolkswagenStiftung (I/83 230). 


\section{References}

Adams EM, Jones AG, Arnold SJ (2005) Multiple paternity in a natural population of a salamander with long-term sperm storage. Molecular Ecology, 14, 1803-1810.

Agresti A (1981) Measures of nominal-ordinal association. Journal of the American Statistical Association, 76, 524-529.

Alcobendas M, Buckley D, Tejedo M (2004) Variability in survival, growth and metamorphosis in the larval fire salamander (Salamandra salamandra): effects of larval brith size, sibship and environment. Herpetologica, 60, 232-245.

Altwegg R, Reyer H-U (2003) Patterns of natural selection on size at metamorphosis in water frogs. Evolution, 57, 872-882.

Amos W, Worthington Wilmer J, Fullard K et al. (2001) The influence of paternal relatedness on reproductive success. Proceeding of the Royal Society of London Series B, 268, 2021-2027.

Birkhead TR, Pizzari T (2002) Postcopulatory sexual selection. Nature Reviews Genetics, 3, 262-273.

Bowcock AM, Ruiz-Linares A, Tomfohrde J, Minch E, Kidd JR, Cavalli-Sforza LL (1994) High resolution of human evolutionary trees with polymorphic microsatellites. Nature, 368, 455-457.

Bos DH, Williams RN, Gopurenko D, Bulut Z, Dewoody JA (2009) Condition-dependent mate choice and a reproductive disadvantage for MHC-divergent male tiger salamanders. Molecular Ecology, 18, 3307-3315.

Caspers BA, Steinfartz S (2011) Preference for the other sex: olfactory sex recognition in terrestrial fire salamanders (Salamandra salamandra). Amphibia-Reptilia, 32, 503-508.

Chandler CH, Zamudio KR (2008) Reproductive success by large, closely related males facilitated by sperm storage in an aggregated breeding amphibian. Molecular Ecology, 17, 15641576.

Clark KR, Warwick RM (1994) Change in Marine Communities: An Approach to Statistical Analysis and Interpretation. PRIMER-E, Plymouth.

Daly M (1978) The cost of mating. The American Naturalist, 112, 771-774.

Dziminski MA, Roberts JD, Simmons LW (2008) Fitness consequences of parental compatibility in the frog Crinia Georgiana. Evolution, 62, 879-886.

Gabor CR, Halliday TR (1997) Sequential mate choice by multiply mating smooth newts: females become more choosy. Behavioural Ecology, 8, 162-166.

Garant D, Dodson JJ, Bernatchez L (2001) A genetic evaluation of mating system and determinants of individual reproductive success in atlantic salmon (Salmo salar L.). Journal of Heredity, 92, 137-145.

Garner TWJ, Schmidt BR (2003) Relatedness, body size and paternity in the alpine newt, Triturus alpestris. Proceedings of the Royal Society of London, Series B, 270, 619-624.

Greven H (1998) Survey of the oviduct of salamandrids with special reference to the viviparous species. Journal of Experimental Zoology, 282, 507-525.

Hansson B, Westerberg L (2002) On the correlation between heterozygosity and fitness in natural populations. Molecular Ecology, 11, 2467-2474.

Hendrix R, Hauswaldt JS, Veith M, Steinfartz S (2010) Strong correlation between cross-amplification success and genetic distance across all members of 'True salamander'(Amphibia:
Salamandridae) revealed by Salamandra salamandra-specific microsatellite loci. Molecular Ecology Resources, 10, 1038-1047.

Hoffman JI, Forcada J, Trathan PN, Amos W (2007) Female fur seals show active choice for males that are heterozygous and unrelated. Nature, 445, 912-914.

Holland B, Rice WR (1999) Experimental removal of sexual selection reverses intersexual antagonistic coevolution and removes a reproductive load. Proceedings of the National Academy of Sciences of the United States of America, 96, 5083-5088.

Jehle R, Sztatecsny M, Wolf JBW, Whitlock A, Hödl W, Burke $T$ (2007) Genetic dissimilarity predicts paternity in the smooth newt (Lissotriton vulgaris). Biology letters, 3, 526-528.

Jennions MD, Petrie M (2000) Why do females mate multiply? A review of the genetic benefits. Biological Reviews, 75, 21-64.

Jones AG (2005) GERUD2.0: a computer program for the reconstruction of parental genotypes from half-sib progeny arrays with known or unknown parents. Molecular Ecology Notes, 5, 708-711.

Jones AG, Adams EM, Arnold SJ (2002) Topping off: a mechanism of first-male sperm precedence in a vertebrate. Proceedings of the National Academy of Sciences of the United States of America, 99, 2078-2081.

Liebgold EB, Cabe PR, Jaeger RG, Leberg PL (2006) Multiple paternity in a salamander with socially monogamous behaviour. Molecular Ecology, 15, 4153-4160.

Liljedal S, Rudolfsen G, Folstad I (2008) Factors predicting male fertilization success in an external fertilizer. Behavioral Ecology and Sociobiology, 62, 1805-1811.

Madsen T, Shine R, Loman J, Hakansson T (1992) Why do female adders copulate so frequently? Nature, 355, 440-441.

Myers EM, Zamudio KR (2004) Multiple paternity in an aggregate breeding amphibian: the effect of reproductive skew on estimated of male reproductive success. Molecular Ecology, 13, 1951-1963.

Park SDE(2001) Trypanotolerance in West African Cattle and the Population Genetic Effects of Selection [Ph.D. thesis] University of Dublin.

Parker GA (1998) Sperm competition and the evolution of ejaculates: towards a theory base. In: Sperm Competition and Sexual Selection (eds Birkhead TR \& Moller AP), pp. 3-54. Academic Press, London.

Rowe G, Beebee TJA, Burke T (1999) Microsatellite heterozygosity, fitness and demography in natterjack toads Bufo calamita. Animal Conservation, 2, 85-92.

Semlitsch RD, Scott DE, Pechmann JHK (1988) Time and size at metamorphosis related to adult fitness in Ambystoma Talpoideum. Ecology, 69, 184-192.

Sever DM, Halliday T, Waights V, Brown J, Davies HA, Moriarty EC (1999) Sperm storage in females of the smooth newt (Triturus v. vulgaris L.): I. ultrastructure of the spermathecae during the breeding season. Journal of Experimental Zoology, 283, 51-70.

Sever DM (2002) Females sperm storage in amphibians. Journal of Experimental Zoology, 292, 165-179.

Sheldon BC (1993) Sexually transmitted disease in birds: occurrence and evolutionary significance. Philosophical Transactions of the Royal Society of London Series B., 339, 491-497.

Sherman CHD, Wapstra E, Uller T, Olsson M (2008) Males with high genetic similarity to females sire more offspring in sperm competition in Peron's tree frog Litoria preonii. Proceedings of the Royal Society of London Series B, 275, 971-978. 
Slatkin M (1974) Hedging one's evolutionary bets. Nature, 250, 704-705.

Slatyer RA, Mautz BS, Backwell PRY, Jennions MD (2012) Estimating genetic benefits of polyandry from experimental studies: a meta-analysis. Biological Reviews, 87, 1-33.

Sluter SM, Kaiser M, Feignoux R, Meyer DR (2007) Reed bunting females increase fitness through extra -pair mating with genetically dissimilar males. Proceedings of the Royal Society of London, Series B, 274, 2865-2871.

Stapleton MK, Kleven O, Lifjeld JT, Robertson RJ (2007) female tree swallows (Tachycineta bicolour) increase offspring heterozygosity through extra pair mating. Behavioural Ecology and Sociobiology, 61, 1725-1733.

Steinfartz S, Küsters D, Tautz D (2004) Isolation of polymorphic tetranucleotide microsatellite loci in the Fire Salamander Salamandra salamandra (Amphibia: Caudata). Molecular Ecology Notes, 4, 626-628.

Steinfartz S, Stemshorn K, Kuesters D, Tautz D (2006) Patterns of multiple paternity within and between annual reproduction cycles of Salamandra salamandra under natural conditions. Journal of Zoology, 268, 1-8.

Steinfartz S, Weitere M, Tautz D (2007) Tracing the first step to speciation - ecological and genetic differentiation of a salamander population in a small forest. Molecular Ecology, 16, 4550-4561.

Tennessen JA, Zamudio KR (2003) Early male reproductive advantage, multiple paternity and sperm storage in an amphibian aggregate breeder. Molecular Ecology, 12, 1567-1576.

Thiesmeier B, Grossenbacher K (2004) Salamandra salamandra - Feuersalamander. In: Handbuch der Reptilien und Amphibien Europas. Schwanzlurche IIB (eds Thiesmeier B \& Grossenbacher K), pp. 1059-1132. Aula Verlag, Wiebelsheim.

Thrall PH, Antonovics J, Bever JD (1997) Sexual transmission of disease and host mating systems: within-season reproductive success. The American Naturalist, 149, 485-506.

Tregenza T, Wedell N (1998) Benefits of multiple mates in the cricket Gryllus bimaculatus. Evolution, 52, 1726-1730.

Tregenza T, Wedell N (2000) Genetic compatibility, mate choice and patterns of parentage: invited review. Molecular Ecology, 9, 1013-1027.

Tregenza T, Wedell N (2002) Polyandrous females avoid costs of inbreeding. Nature, 415, 71-73.

Trivers RL (1972) Parental investment and sexual selection. In Sexual Selection and the Descent of Man (ed Campbell B), pp. 136-179. Aldine Pub. Co. Chicago.

Waage JK (1979) Dual function of the damselfly penis: sperm removal and transfer. Science, 203, 916-918.

Wigby S, Chapman T (2004) Sperm competition. Current Biology, 14, R100-R103.

Zeh JA, Zeh DW (1997) The evolution of polyandry II: postcopulatory defenses against genetic compatibility. Proceedings of the Royal Society of London Series B, 264, 69-75.

\section{Data accessibility}

Microsatellite data of all females and offspring used for this study are available at DRYAD doi:10.5061/dryad. jp203.

This study is part of the postdoc project of B.A.C. on speciation mechanisms in salamanders conducted in the group of S.S. B.A.C. has a research interest in animal behaviour and especially in olfaction. E.T.K. is interested in behavioural ecology and cooperates on the salamander project with B.A.C. and S.S. R.H. is currently a $\mathrm{PhD}$ student in the group of S.S. and is working on genetic and demographic analyses of salamander populations. S.S. has a strong interest in molecular ecology, population ecology and speciation of amphibians and reptiles. M.K. is a theoretical evolutionary biologist interested in the population genetics of adaptation and speciation. O.R. is a PhD student in the group of Computational Genomics now located the University of Giessen. K.R. did her practical state exam in this project and is now a high school teacher.

\section{Supporting information}

Additional supporting information may be found in the online version of this article.

Data S1 PCR-multiplex combinations and specific amplification procedures of $18 \mathrm{~S}$. salamandra specific microsatellite loci.

Data S2 Allele-frequencies (18 loci) of the Kottenforst population, based on 1100 adults captured from 2007-2011.

Data S3 Exclusion probabilities of applied loci.

Data S4 Proportion (\%) of correctly found sires in simulated offspring arrays with 4 and 10 larvae, respectively.

Data S5 Factors influencing larval mass and size at the time of birth/deposition.

Data S6 Internal relatedness and mean observed heterozygosity of deposited larvae.

Data S7 Unfertilized eggs/non-viable embryos. 